\title{
HEMORRAGIA DIGESTIVA BAIXA DEVIDO A MELANOMA MALIGNO METASTÁTICO
}

\section{LOWER GASTROINTESTINAL BLEEDING DUE TO METASTATIC MALIGNANT MELANOMA}

\author{
Orlando Ribeiro Prado Filho, TCBC-PR ${ }^{1}$ \\ Thayse Gonçalves de Lima \\ Orlando Gonçalves Monteiro ${ }^{3}$
}

\section{INTRODUÇÃO}

O melanoma maligno é a causa mais comum de lesão metastática no trato gastrointestinal (GI), representando cerca de $33 \%$ de todos os tumores malignos metastáticos do tubo digestivo ${ }^{1}$. As manifestações mais freqüentes são: dor abdominal, hemorragia macroscópica ou microscópica, náusea/ vômito, tumor abdominal e obstrução intestinal ${ }^{1}$.

O objetivo deste trabalho é apresentar o caso clínico, diagnóstico e tratamento de um paciente com quadro de hemorragia digestiva baixa decorrente de metástase de melanoma maligno no intestino delgado.

\section{RELATO DO CASO}

Paciente do sexo masculino, branco, com idade de 56 anos, foi atendido por queixa de dor abdominal difusa, diarréia com sangue e astenia. Relatou que há cinco anos fora submetido à exérese de lesão na pele. Ao exame físico apresentava-se com pressão arterial sistêmica de 90/ $60 \mathrm{mmHg}$, freqüência cardíaca de 110 batimentos por minuto, palidez e dor abdominal difusa moderada, sem sinais de irritação peritoneal. Foi internado e os exames laboratoriais mostraram anemia $(\mathrm{Hb}=8,6 \mathrm{~g} \% \mathrm{e} \mathrm{Ht}=25 \%)$. Foi submetido a exame endoscópico digestivo alto (normal) e colonoscopia, que mostrou doença diverticular difusa, sem sangramento ativo. Durante a internação o paciente apresentou novo sangramento digestivo baixo, sendo submetido à celiotomia exploradora com o achado de neoplasia no íleo há $35 \mathrm{~cm}$ da válvula ileocecal, sem linfoadenopatia regional ou metástase a distância. Foi realizada enterectomia segmentar de $15 \mathrm{~cm}$ de extensão do íleo com anastomose primária. A peça cirúrgica foi (Figuras 1 e 2) encaminhada para exame anatomopatológico, que revelou melanoma maligno. O paciente recebeu alta em boas condições gerais no décimo dia pós-operatório.

\section{DISCUSSÃO}

O melanoma maligno tem uma grande predileção para desenvolver metástases no trato $\mathrm{GI}^{2}$.O intestino delgado esta comprometido em $35 \%$ dos pacientes ${ }^{3}$, sendo o íleo afetado em cerca $41 \%$ e o jejuno em cerca de $85 \%$ dos $\operatorname{casos}^{1}$. A forma mais comum de metástase é com múltiplos implantes na submucosa, que tendem a crescer em direção à luz do tubo digestivo ${ }^{1}$.

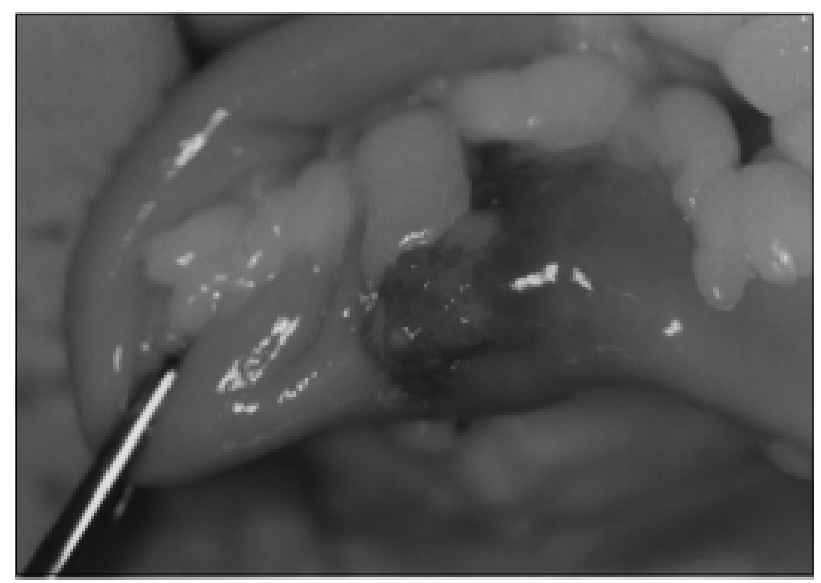

Figura 1 - Fotografia do aspecto macroscópico externo do melanoma maligno metastático no íleo, ainda na cavidade abdominal .

1. Professor Assistente da Área de Clínica Cirúrgica do Departamento de Medicina da Universidade Estadual de Maringá (UEM) - Maringá, PR.

2. Acadêmica do Curso de Medicina da UEM - Maringá, PR.

3. Médico Residente de Cirurgia Geral da Área de Clínica Cirúrgica do Departamento de Medicina da UEM - Maringá, PR.

Recebido em 08/11/1999

Aceito; para publicação em 22/05/2001

Trabalho realizado na Área de Clínica Cirúrgica do Departamento de Medicina da Universidade Estadual de Maringá Maringá, PR. 


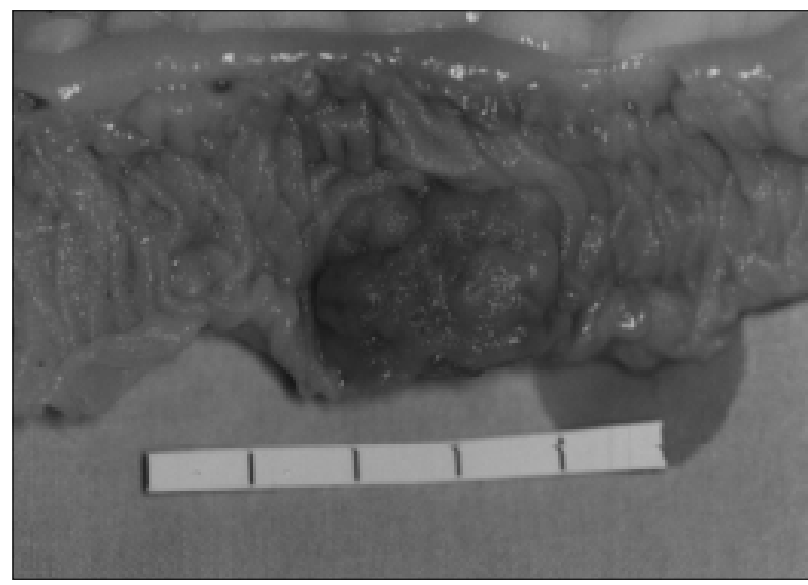

Figura 2 - Fotografia do aspecto macroscópico interno do melanoma maligno metastático no íleo, após abertura longitudinal da peça operatória.

O intestino delgado é fonte do sangramento digestivo baixo em cerca de $3 \%$ a $5 \%$ dos casos relatados, sendo as neoplasias causas raras. Nos caso em que o sangramento tenha cessado ou diminuído de forma significante o exame diagnóstico de escolha é a colonoscopia, devido à sua acurácia diagnóstica e capacidade terapêutica ${ }^{4}$. Outros exames utilizáveis são a cintilografia com tecnécio 99m (inje- tado diretamente na corrente sangüínea ou em hemácias marcadas), em casos de sangramentos intermitentes, e a angiografia mesentérica seletiva para sangramentos de 0,5 a $1,0 \mathrm{ml} / \mathrm{minuto}$ de vazão mínima .

As indicações operatórias são: perda aguda maior que $1.500 \mathrm{ml}$ e continuidade do sangramento, necessidade de transfusão de $2.000 \mathrm{~mL}$ em $24 \mathrm{~h}$, sangramento contínuo por $72 \mathrm{~h}$ e ressangramento significante ocorrendo dentro do período de uma semana ${ }^{4}$.

No caso apresentado excluiu-se hemorragia digestiva alta e fez-se a investigação do tubo digestivo baixo onde encontrou-se doença diverticular difusa. Como o paciente voltou a aparência sangramento durante o período de internamento preferiu-se não prosseguir com outros exames possíveis, e inclinou-se para o tratamento operatório de urgência, como assim é indicado.

Estudos recentes demonstram uma sobrevida maior para os pacientes em que se foi capaz de fazer a completa retirada da doença neoplásica metastática, o que tem renovado o interesse na ressecção do melanoma no trato $\mathrm{GI}^{5}$.

Concluindo, o sangramento digestivo baixo é um problema complexo que requer investigação disciplinada para que se possa determinar a melhor forma de tratamento. A ressecção cirúrgica completa do melanoma metastático no intestino delgado pode ser realizada de forma segura, o que pode propiciar aos pacientes um tempo de sobrevida maior.

\begin{abstract}
A case of lower gastrointestinal bleeding due to metastatic malignant melanoma in a man, in which the final diagnosis was made only on surgery, is reported. The patient underwent a segmentary enterectomy with primary anastomosis and he was discharged on tenth postoperative day.
\end{abstract}

Key Words: Gastrointestinal bleeding; Melanoma; Metastasis.

\section{REFERÊNCIAS}

1. Berger AC, Buel JF, Venzon D, Barker AR, Libutti SK - Manangement of symptomatic malignant melanoma of the gastrointestinal tract. Ann Surg Oncol , 1999; 6:155-160.

2. Elsayed AM, Albahra M, Nzeako UC, Sobin LH - Malignant melanomas in the small intestine: a study of 103 patients. Am J Gastroenterol, 1996; 91:1001-1006.

3. Reitgen DS, Thompson W, Garbutt J, Seigler HF - Radiologic, endoscopic, and surgical considerations of melanoma metastatic to the gastrointestinal tract. Surgery, 1984; 95:635-639.

4. Vernava III AM, Moore BA, Longo WE, Johnson FE - Lower gastrointestinal bleeding. Dis Colon Rectum , 1998; 40:846-858.
5. Branum GD, Sigler HF - Role of surgical intervention in the manangement of intestinal metastases from malignant melanoma. Am J Surg 1991; 162:428-431.

Endereço para Correspondência

Dr. Orlando Ribeiro Prado Filho

Rua Silva Jardim, 560 - Centro

87013-010 - Maringá, PR 